\title{
Inertial waves in rapidly rotating flows: a dynamical systems perspective
}

\author{
Juan M Lopez ${ }^{1}$ and Francisco Marques ${ }^{2}$ \\ ${ }^{1}$ School of Mathematical and Statistical Sciences, Arizona State University, \\ Tempe AZ 85287, USA \\ ${ }^{2}$ Departament de Física Aplicada, Univ. Politècnica de Catalunya, Barcelona \\ 08034, Spain
}

\begin{abstract}
.
An overview of recent developments in a wide variety of enclosed rapidly rotating flows is presented. Highlighted is the interplay between inertial waves, which have been predicted from linear inviscid considerations, and the viscous boundary layer dynamics which result from instabilities as the nonlinearities in the systems are increased. Further, even in the absence of boundary layer instabilities, nonlinearity in the system often leads to complicated interior flows due to subcritical instabilities, Eckhaus bands and heteroclinic dynamics. The ensuing spatio-temporally complex dynamics are analysed in terms of equivariant dynamical systems, providing a general perspective for the wide range of dynamics involved.

PACS numbers: $\quad 47.32 . \mathrm{Ef}, 47.20 . \mathrm{Ky}, 47.52 .+\mathrm{j}$
\end{abstract}

Submitted to: Phys. Scr. 


\section{Introduction}

Rapidly rotating flows are generically in solid body rotation due to the strong restoring Coriolis force. In this sense, rapid rotation means that the rotation period is much shorter than the viscous time scale of the problem. In practice a ratio of about $10^{4}$ is sufficient to be in the rapid rotation regime. This can be quantified in terms of $\omega_{0}=\Omega_{0} R^{2} / \nu$, where $\Omega_{0}$ is the rotation rate, $R$ is a length scale, and $\nu$ is the kinematic viscosity. The governing parameter $\omega_{0}$ can be interpreted as a Reynolds number, or an inverse Ekman number; it is a ratio of the viscous time and the rotation period. Enclosed rapidly rotating flows can be driven away from solid body rotation by various perturbations to the system. One way is via steady differential rotation of different parts of the enclosure boundaries. Here, the interior remains in solid body rotation at the mean rotation rate of the boundaries [3], and the adjustment between the interior rotating flow and the differentially rotating boundaries is accomplished in boundary layers which scale as inverse powers of $\omega_{0}$, with the powers depending on how the boundaries are oriented with respect to the mean rotation axis [40]. As the rotation becomes more rapid (increasing $\omega_{0}$ ), the boundary layers become thinner and more intense and at some critical $\omega_{0}$ instabilities ensue. Turbulent mixing is confined by the Coriolis force to the viscous boundary layers, except when the temporal spectrum of the instabilities has frequencies less than twice the mean rotation rate, in which case energy is transported into the interior in shear layers aligned along the directions associated with the characteristics of the hyperbolic partial differential equation obtained by linearizing about the mean solid body rotation of the interior flow and ignoring viscous effects in the interior [10]. In order to drive boundary layers via steady differential rotation to such instabilities, the differential rotation needs to be sufficiently large, quantified via the ratio $\delta \omega=\Delta \Omega / \Omega_{0}$, where $\Delta \Omega$ is the differential in the rotation of the boundary. For $\omega_{0} \sim 10^{5}, \delta \omega$ tends to be of order $10^{-1}$ or larger for such instabilities, and this typically renders the nonlinear terms in the Navier-Stokes equations non-negligible, and often there are nonlinear modifications to the mean solid body rotation interior flow.

Another way to drive the flow away from solid body rotation is to apply a body force in addition to the Coriolis term due to the mean rotation. A typical example is precessing flows, where the angular velocity of the container is misaligned with the cylinder axis. In these cases the interior flow is driven away from solid body and a non-negligible meridional overturning flow is forced. When the precession frequency is less than $2 \Omega_{0}$, this meridional flow has similarities with low-order Kelvin eigenmodes (the eigenmodes of the Euler equations linearized about solid body rotation in the cylinder). However, even when the precession frequency is greater than $2 \Omega_{0}$, similar meridional flows are driven, which have no interpretation in terms of Kelvin modes.

To drive perturbations with frequencies less than twice the background rotation frequency, whilst keeping $\omega_{0}$ large and $\delta \omega$ small, one can drive the differential rotation harmonically with the desired frequency and very small amplitude. This allows one to interrogate the resulting flow within the perspective of the linear inviscid theory [10]. However, this perspective needs to be considered with some care, as the reduction from the Navier-Stokes equations to the Euler equations is a singular perturbation which does not allow all the boundary conditions governing the flow to be considered. Typically, the no slip boundary conditions that are imposed on the Navier-Stokes description of the problem are reduced to the no penetration boundary conditions in the Euler description. One consequence is that since differential equations admit a 
multitude of solutions and the boundary and initial conditions restrict which of those are permissible for a given flow configuration, the solutions of the Euler equations are typically not solutions of the Navier-Stokes equations. In some cases, the two solutions sets are similar, differing in the presence of thin boundary layers in the Navier-Stokes solutions with the two agreeing to some degree away from the boundary layer regions. However, more typically, the thin boundary layers become unstable at large $\omega_{0}$ leading to turbulence as $\omega_{0} \rightarrow \infty$ and the flow in the limit $\omega_{0} \rightarrow \infty$ (NavierStokes) ends up being very different to the flow at the limit $\omega_{0}=\infty$ (Euler). Despite over a century of study, the saturation amplitude of instabilities, the conditions for the apparition of intermittent cycles, the type of turbulence and its associated spectra, and the clarification of the bifurcation sequences leading to turbulence are all still open questions in rapidly rotating flows $[15,20]$.

\section{Governing equations for enclosed cylinder flows}

To be specific, we discuss the case of a cylindrical geometry, where several recent experimental, theoretical and numerical studies have been reported. The apparatus consists of a cylinder of height $H$ and radius $R$ filled with an incompressible fluid of kinematic viscosity $\nu$. All variables are non-dimensionalized using the cylinder radius $R$ as the length scale and the viscous time $R^{2} / \nu$ as the time scale. The aspect ratio $\Gamma=H / R$ is one of the non-dimensional parameters of the problem. The governing equations are written using cylindrical coordinates $(r, \theta, z)$, with the origin $O$ at the centre of the cylinder and the $z$ direction aligned with the cylinder axis:

$$
\partial_{t} \boldsymbol{v}+(\boldsymbol{v} \cdot \nabla) \boldsymbol{v}=-\nabla p+\boldsymbol{b}+\Delta \boldsymbol{v}, \quad \nabla \cdot \boldsymbol{v}=0 .
$$

The body force $\boldsymbol{b}$ depends on the frame of reference used, and typically will include the Coriolis force. The velocity field in cylindrical coordinates is $\boldsymbol{v}=(u, v, w)$, the vorticity field is $\nabla \times \boldsymbol{v}=(\xi, \eta, \zeta)$, and their scalar product is the helicity $\mathcal{H}=\boldsymbol{v} \cdot(\nabla \times \boldsymbol{v})$. It is convenient to expand the velocity field in its azimuthal Fourier components $\boldsymbol{v}_{m}$, where $m$ is the azimuthal wave number. The kinetic energies of these components, $E_{m}$, is very useful in describing the flow properties.

The boundary conditions are no slip on the cylinder walls, and depend on the imposition of differential rotation and/or temporal forcing on parts of the cylinder. The cylinder geometry is invariant under rotations and reflections about the cylinder axis, as well as reflection about the mid-plane orthogonal to the axis, $z=0$. These orthogonal transformations generate the symmetry group of the cylinder, $O(2) \times Z_{2}$. For a specific problem, the body force and the boundary conditions may not be equivariant under the $O(2) \times Z_{2}$ symmetry group, but only equivariant under a subgroup. It is well known that the symmetries of the problem strongly influence the dynamics and the types of bifurcations and instabilities the system may experience as parameters are varied [6].

\section{Theoretical background on linear inviscid rotating flows}

The theoretical study of inertial waves considers the cylindrical container in solid body rotation about its axis, with angular velocity $\Omega_{0}$, characterised by the non-dimensional number $\omega_{0}=\Omega_{0} R^{2} / \nu$. In the frame of reference rotating with the cylinder, the body force in (1) is the Coriolis force, $\boldsymbol{b}=-2 \boldsymbol{\omega}_{0} \times \boldsymbol{v}$, and the boundary conditions for the velocity field are homogeneous, $\boldsymbol{v}=\mathbf{0}$, on the cylinder boundary. The non-dimensional 
angular velocity is $\boldsymbol{\omega}_{0}=\omega_{0} \hat{\boldsymbol{z}}$. Linearizing about solid body rotating and neglecting viscous effects, (1) is reduced to

$$
\partial_{t} \boldsymbol{v}+2 \boldsymbol{\omega}_{0} \times \boldsymbol{v}=-\nabla p, \quad \nabla \cdot \boldsymbol{v}=0,
$$

with no penetration boundary conditions (zero normal velocity on the cylinder boundary).

\subsection{Plane waves, rays and wave beams}

The usual approach is to begin by looking for plane wave solutions of the differential equation (2), irrespective of the boundary conditions:

$$
\boldsymbol{v}=\boldsymbol{A} \exp \{\mathrm{i}(\boldsymbol{k} \cdot \boldsymbol{r}-\sigma t)\} \text { and } p=a \exp \{\mathrm{i}(\boldsymbol{k} \cdot \boldsymbol{r}-\sigma t)\},
$$

where $\sigma$ is the wave frequency, $\boldsymbol{k}$ the wave vector, and $\boldsymbol{A}$ and $a$ are the complex wave amplitudes. Substituting these into (2) gives the dispersion relation $\sigma(\boldsymbol{k})$ :

$$
\sigma^{2} k^{2}=\left(2 \boldsymbol{k} \cdot \boldsymbol{\omega}_{0}\right)^{2} \text { or } \sigma= \pm 2 \omega_{0} \cos \beta, \boldsymbol{k} \cdot \boldsymbol{\omega}_{0}=k \omega_{0} \cos \beta,
$$

where $\beta$ is the angle between the wave vector $\boldsymbol{k}$ and the cylinder rotation axis $\boldsymbol{\omega}_{0}$. The phase and group velocities are given by

$$
\boldsymbol{c}_{\phi}=\frac{\sigma}{k^{2}} \boldsymbol{k} \text { and } \boldsymbol{c}_{g}=\nabla_{k} \sigma=\frac{\sigma}{k^{2}\left(\boldsymbol{k} \cdot \boldsymbol{\omega}_{0}\right)} \boldsymbol{k} \times\left(\boldsymbol{\omega}_{0} \times \boldsymbol{k}\right) .
$$

Their moduli are

$$
c_{\phi}=\frac{\sigma}{k} \text { and } c_{g}=\frac{\sigma}{k} \tan \beta .
$$

The direction of wave propagation, $\boldsymbol{c}_{g}$, is orthogonal to the wave vector, therefore $\beta$ is the angle that $\boldsymbol{c}_{g}$ makes with planes orthogonal to the cylinder axis. This angle is independent of the wave number $k$, and depends only on the ratio of the wave frequency to the solid body rotation frequency: $\cos \beta=\sigma / 2 \omega_{0}$. Plane waves only exist for frequencies less than twice the solid body rotation frequency. The directions of propagation of the plane inertial waves from a given point form a cone with its vertex at the given point. These directions are the characteristics of the hyperbolic equation (2). The details of the structure of the cone depend on the nature of the perturbation [29].

As the dispersion relation does not depend on the wave number (it depends on the direction of $\boldsymbol{k}$ but not on its modulus $k$ ), for a given wave frequency there are many (an infinite continuum) of plane wave solutions. Any linear combination of plane waves, either discrete (Fourier series) or continuous (Fourier integral), is a solution of the inviscid linear equations: it is a wave packet. However, since the individual plane waves do not satisfy the no penetration boundary conditions, additional conditions on the coefficients of the linear combination constituting the wave packet need to be imposed in order to satisfy the boundary conditions.

If a perturbation with a given frequency $\sigma$ is introduced at a given point in space, the perturbation propagates along the cone of characteristics from that point, with a spatial extent transverse to the propagation direction given by the spatial extent of the perturbation. These are called wave beams. If the perturbation has zero spatial extent (a Dirac delta function), the propagating perturbation is called a ray. It is a straight line, a characteristic of the hyperbolic equation (2), along which the discontinuity (delta function) propagates. When wave beams or rays reach the domain boundary, they are reflected and return back into the interior of the domain along the 
characteristic directions. The original beam and its successive reflections result in a complicated interference pattern, except when the beams or rays retrace themselves, resulting in a simple pattern.

If the perturbation has more than one frequency, there are several cones of characteristics, one for each frequency. Moreover, as inertial waves are dispersive waves, the multi-frequency beams broaden with time. In this case the resulting pattern may be very complex.

Real flows are viscous and viscosity has two main effects on inertial wave beams: viscous damping and viscous spreading. The attenuation factor for a planar monochromatic inertial wave as it travels a distance $x=c_{g} t$ along the characteristic can be written as $[7]$

$$
C_{d}=e^{-k^{2} t}=e^{-\ell^{2} k^{3} x},
$$

with $\ell=1 / \sqrt{\sigma \tan \beta} ; \ell^{*}=\sqrt{\nu} / \sqrt{\sigma^{*} \tan \beta}$ is a dimensional viscous length scale and $\sigma^{*}$ is the dimensional wave frequency. The beam components with high wave numbers $k$ are preferentially attenuated and disappear from the beam. Eventually the beam only retains the components with smaller $k$ (larger wavelengths). Of course, additional factors must also be considered, such as geometric focusing effects (for non-planar waves) that may enhance the amplitude of the waves [8], in contrast to focusing onto wave attractors due to reflections off boundaries at oblique angles to the rotation axis [28].

The thickness of the wave beam $\delta(x)$ changes with the distance $x$ travelled from the perturbation source via viscous spreading. This results in a corresponding change in the velocity and vorticity amplitudes along the beam. For an inertial wave beam generated by a linear source, these changes are [7]

$$
\frac{\delta(x)}{\ell} \sim\left(\frac{x}{\ell}\right)^{1 / 3}, \quad \boldsymbol{v}(x) \sim\left(\frac{\ell}{x}\right)^{1 / 3}, \quad(\nabla \times \boldsymbol{v})(x) \sim\left(\frac{\ell}{x}\right)^{2 / 3} .
$$

\subsection{Kelvin eigenmodes}

Up to now, we have discussed inertial waves irrespective of the boundary conditions. One can also look for solutions of (2) satisfying no penetration boundary conditions. The result depends strongly on the geometry considered, and we will discuss only the cylindrical case. Using cylindrical coordinates $(r, \theta, z) \in[0,1] \times[0,2 \pi] \times[-\Gamma / 2, \Gamma / 2]$, where $\Gamma=H / R$ is the aspect ratio and the origin is at the centre of the cylinder, we seek solutions of the form

$$
\boldsymbol{v}=\exp \{\mathrm{i}(m \theta-\sigma t)\} \boldsymbol{w}(r, z) \text { and } p=2 \omega_{0} \exp \{\mathrm{i}(m \theta-\sigma t)\} \Phi(r, z) .
$$

Substitution into (2) results in the equation for $\Phi$ :

$$
r^{2} \Phi_{r r}+r \Phi_{r}-m^{2} \Phi+r^{2}\left(1-\frac{1}{F^{2}}\right) \Phi_{z z}=0, \quad F=\frac{\sigma}{2 \omega_{0}},
$$

with boundary conditions

$$
\begin{aligned}
& \Phi_{z}=0 \text { at } z= \pm \Gamma / 2, \\
& F \Phi_{r}+\frac{m}{r} \Phi=0 \text { at } r=1,
\end{aligned}
$$

and regularity at $r=0$. This eigenvalue problem can be solved by separation of variables, and the solutions are the Kelvin eigenmodes, enumerated by three integer indices $(j, m, n)$ corresponding to the wave numbers in the $(r, \theta, z)$ directions:

$$
\Phi_{j m n}(r, z)=C J_{m}\left(\delta_{j m n} r\right) \cos n \pi\left(\frac{z}{\Gamma}+\frac{1}{2}\right),
$$


where $\delta_{j m n}(\Gamma), j>0$, are the roots of

$$
\delta J_{m}^{\prime}(\delta)+m \sqrt{1+\left(\frac{\Gamma \delta}{n \pi}\right)^{2}} J_{m}(\delta)=0 .
$$

The velocity field can be reconstructed from $\Phi . F, \beta$ and $\sigma$ are functions of $\delta, \Gamma$ and $\omega_{0}$ :

$$
F_{j m n}=\cos \beta_{j m n}=\left[1+\left(\frac{\Gamma \delta_{j m n}}{n \pi}\right)^{2}\right]^{-1 / 2}, \quad \sigma_{j m n}=2 \omega_{0} F_{j m n} .
$$

The frequency spectrum $\sigma_{j m n}$ densely fills the interval $\left[0,2 \omega_{0}\right]$. Kelvin eigenmodes are also damped by viscosity, with an attenuation factor of the form $C_{d}=e^{-D_{j m n} t}$, where the coefficient $D_{j m n}$ is complicated to estimate [10, 45], but can be approximated by $D_{j m n} \sim(n \pi / \Gamma)^{2}+\delta_{j m n}^{2}$. For large $j, \delta_{j m n} \sim j \pi / 2$, and so in any linear combination of Kelvin modes the components with large values of $n$ and $j$ are preferentially attenuated and disappear. With respect to the azimuthal wave number $m$, [44] showed that the Kelvin modes with large $m$ are confined near the sidewall, where the boundary layers are dominant. As a result, only Kelvin eigenmodes with small values of $(j, m, n)$ will be relevant.

Any linear combination of the $(j, m, n)$ Kelvin eigenmodes is also a solution of the inviscid linearized equations with no penetration boundary conditions. However, the Kelvin eigenmodes do not satisfy the physical no slip boundary condition. Adjusting the coefficients of the linear combination of Kelvin eigenmodes so that the no slip boundary condition is satisfied is not enough, because the boundary layers that form on the cylinder walls are not accounted for. There are complicated ways to overcome this problem, but the singularity of the inviscid limit leads to ad hoc methods having poor convergence properties [45].

We have seen that local perturbations in a rapidly rotating flow result in beams of inertial waves emanating from the local source. When the perturbation is global, acting over the whole domain (for example, via a body force such as in precessing flows), the flow solutions often resemble Kelvin eigenmodes in the bulk. However, if the perturbation has a specific frequency, $\omega_{f}$, the solution also has this frequency. At sufficiently small $\omega_{0}$, the base state of the problem is unique and synchronous with the forcing. There are Kelvin modes with frequencies arbitrarily close to $\omega_{f}$, but typically they have large $k$ and are rapidly damped out. The base state in the bulk often resembles $(j, m, n)$-Kelvin modes with low $k$, but with the imposed frequency instead of the frequency $\sigma_{j m n}$ predicted by the linearized Euler equations. The solutions of the linearized Euler equations would only approximate the solution of the full Navier-Stokes equations in the limit of $\omega_{0} \rightarrow \infty$. However, at large $\omega_{0}$, the associated nonlinear dynamics completely overcomes the Kelvin modes, and the flow is fully turbulent. The limit $\nu \rightarrow 0$ is singular, and the validity of approximations based on Kelvin mode expansions is very limited, typically useful only at moderate $\omega_{0}$, before the nonlinearities dominate the dynamics.

\section{Rotating cylinder flows with finite viscosity and nonlinearity}

We now provide an overview, from our own perspective, of a variety of rotating cylinder flows. The flows are in finite cylinders, so that viscous boundary layers are always present, and at slow enough rotation rates, there exists a unique basic state [39]. The primary issue to be address here is what is the fate of this basic state as the rotation 
(a)

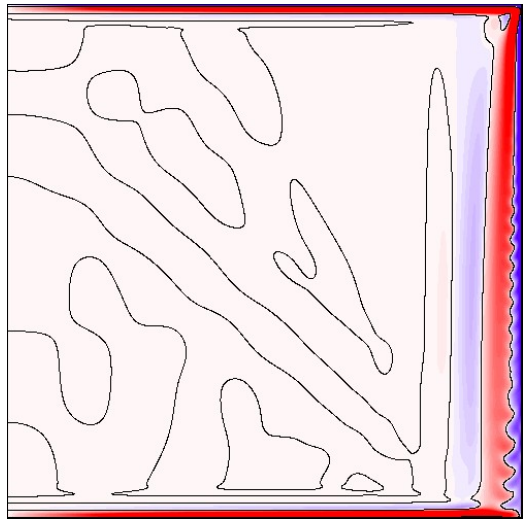

$(b)$

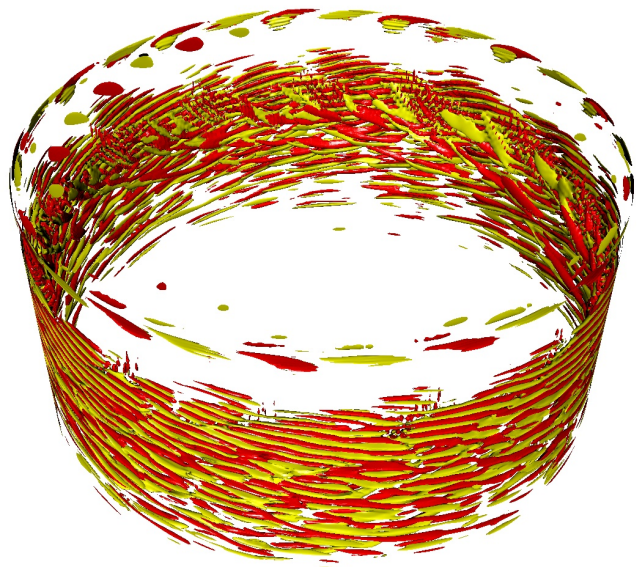

Figure 1. Snapshot of $(a)$ azimuthal vorticity $\eta$ of a quasiperiodic state at $\omega_{0}=4.2 \times 10^{4}, \delta \omega=0.50$ and $\Gamma=1$, compututing in the axisymmetric subspace, and $(b)$ sosurfaces of the non-axisymmetric components of the azimuthal vorticity $\eta-\eta_{0}$ ( $\eta_{0}$ is the axisymmetric component), of the spatio-temporally complex state at $\omega_{0}=5 \times 10^{4}, \delta \omega=0.50$ and $\Gamma=1$ (from [23]).

rate is increased, and how does the linear inviscid theory summarised in the previous section fit into the viscous nonlinear realm.

\subsection{Steady differential rotation}

In [21], a wide variety of differentially rotating cylinder flows were considered, but $\omega_{0}$ was kept relatively small and only axisymmetry flows were considered (due to computational constraints at the time). Inertial wave beams were observed emanating from the junctions where the cylinder sidewall and endwalls meet, but they were not explored at the time. More recently, [23] explored in some detail one of the cases studied in [21], where the differential rotation was driven by the top endwall rotating faster than the rest of the cylinder. The flow is governed by (1), where in the laboratory frame $\boldsymbol{b}=\mathbf{0}$, and the boundary conditions are:

$$
\begin{aligned}
& \boldsymbol{v}=\left(0, r \omega_{0}(1+\delta \omega), 0\right) \text { at the top endwall } z=\Gamma / 2, \\
& \boldsymbol{v}=\left(0, \omega_{0}, 0\right) \text { at the sidewall } r=1, \\
& \boldsymbol{v}=\left(0, r \omega_{0}, 0\right) \text { at the bottom endwall } z=-\Gamma / 2,
\end{aligned}
$$

where $\delta \omega$ measures the differential rotation. This differential rotation results in a large scale meridional circulation, where the flow in the Ekman boundary layer on the faster rotating endwall drives flow into a sidewall boundary layer, which when the differential rotation is very small is of Stewartson type. Some of the flow in the sidewall layer is turned into a boundary layer on the bottom endwall from where there is a slow effusive flow back into the top Ekman layer through the interior. The interesting dynamics occurs when $\delta \omega \sim O\left(10^{-1}\right)$ and larger. The sidewall layer changes its structural characteristics, developing an inflectional radial profile in the axial velocity, and undergoes a number of distinct instabilities, some of which occur concurrently. These were first observed experimentally [13], but the experiments were not able to fully explore the nonlinear dynamics involved. When the simulations of [23] were 
(a) QP:
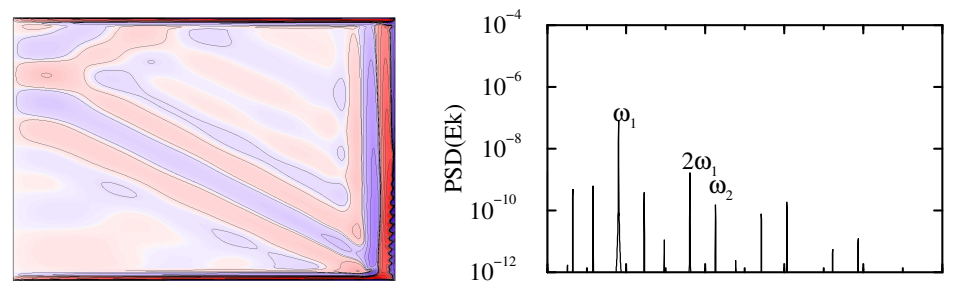

(b) QP2:

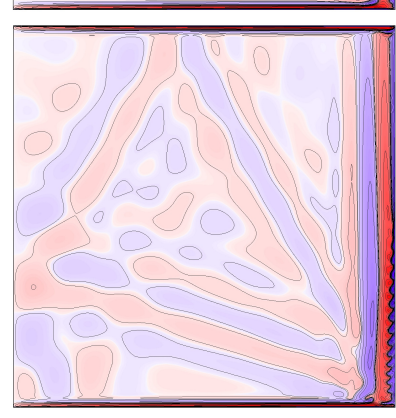

$10^{-12}$

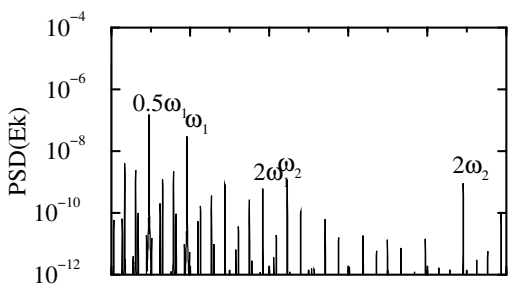

(c) QP4:
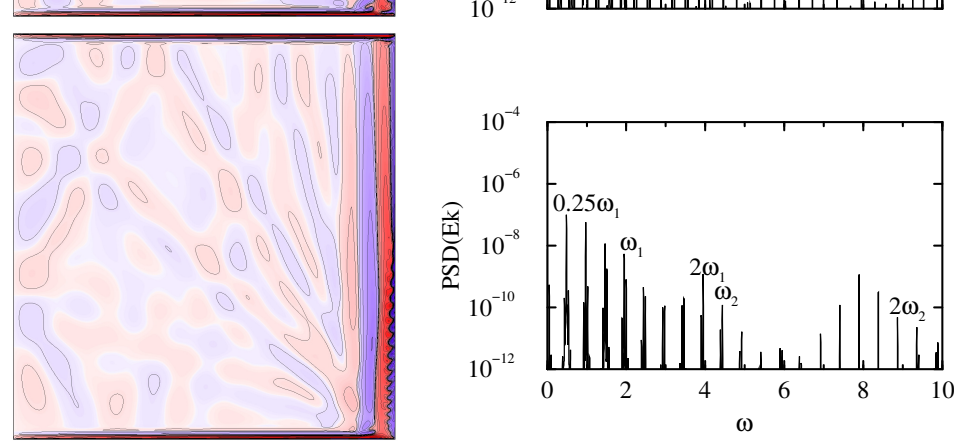

Figure 2. Snapshots of azimuthal vorticity $\eta$ (left) and the corresponding power spectral densities of the kinetic energy (right), at $\omega_{0}=10^{5}$ for $(a)$ QP at $\delta \omega=0.105, \Gamma=0.6875,(b) \mathrm{QP} 2$ at $\delta \omega=0.12, \Gamma=1$, and $(c)$ QP4 at $\delta \omega=0.11$, $\Gamma=1.0625$. Ten cubically spaced contour levels are in the range $\eta \in[-5,5]$.

restricted to the axisymmetric subspace, as in [21], some of the sidewall instabilities had frequencies less than $2 \Omega_{0}$ which drove wave beams into the interior from the junction where the cylinder sidewall and bottom endwall meet. Figure 1(a) shows an example of such a flow. However, the unrestricted three-dimensional simulations of [23] showed that the primary instabilities are not axisymmetric and lead to complicated flows with high azimuthal wave numbers with the turbulence confined to the sidewall boundary layer. This is consistent with the theoretical considerations in section $\S 3.1$ concerning the damping of high wave number inertial waves. Figure 1(b) shows an example of such a flow, which has many of the features observed in the experimental flow of [13].

In order to address the question concerning the role of the discontinuous boundary condition at the junction where the faster rotating endwall meets the slower rotating sidewall, [12] considered the differentially rotating split cylinder, where the cylinder is split into two halves at mid-height, and one half rotates faster than the other. The governing equations in the laboratory frame are (1) with $\boldsymbol{b}=\mathbf{0}$, and the boundary 
(a) $\mathrm{RW}_{1}$

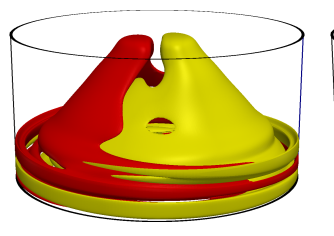

(b) $\mathrm{RW}_{2}$

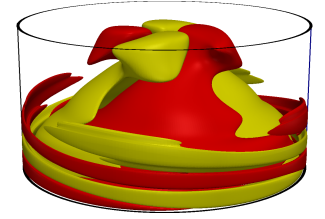

(c) $\mathrm{RW}_{3}$

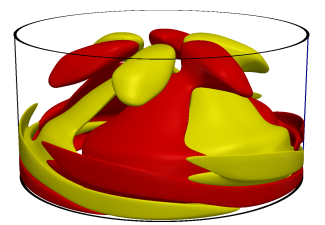

(d) $\mathrm{RW}_{4}$

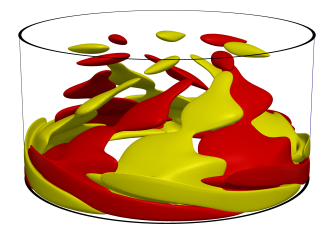

Figure 3. Isosurfaces of $w-w_{0}$, at levels \pm 0.001 , of rotating waves $\mathrm{RW}_{m}$ with azimuthal wave number $m$ as indicated, all for $\omega_{0}=10^{4}, \delta \omega=0.29$ and $\Gamma=1$.

conditions are:

$$
\begin{aligned}
& \boldsymbol{v}=\left(0, r \omega_{0}(1+\delta \omega), 0\right) \text { at the top endwall } z=\Gamma / 2, \\
& \boldsymbol{v}=\left(0, \omega_{0}(1+\delta \omega), 0\right) \text { at the top sidewall } r=1, z>0, \\
& \boldsymbol{v}=\left(0, \omega_{0}, 0\right) \text { at the bottom sidewall } r=1, z<0, \\
& \boldsymbol{v}=\left(0, r \omega_{0}, 0\right) \text { at the bottom endwall } z=-\Gamma / 2 .
\end{aligned}
$$

The endwall boundary layers are similar to those in [23], and the sidewall layers also have some features in common, but at low $\delta \omega$ the roles of the $\omega_{0}{ }^{-1 / 3}$ and $\omega_{0}{ }^{-1 / 4}$ scaling of the sidewall boundary layer thickness changes; [42] showed that the role of these scaling depends on the axial location of the split in the cylinder. The axisymmetric simulations of [12] showed that the discontinuity at the split plays no significant dynamic role, other than to provide the differential rotation that drives a large scale meridional flow, as in [23], and that it is the nonlinear sidewall layer instability that leads to unsteady flows that are able to drive inertial waves into the interior. They considered larger $\omega_{0}$ flows over an extensive range of $\delta \omega$, observed a period doubling sequence of bifurcations which resulted in quasiperiodic flows that drove inertial waves along cones with different cone angles associated with the various frequencies, resulting in a complicated internal flow criss-crossed by an intricate network of conical shear layers. Figure 2 shows snap-shots of the azimuthal vorticity contours of three quasiperiodic states. The first, QP, is a mixed mode of two limit cycles, one with frequency $\omega_{1}$ and the other with $\omega_{2}$ which come about as distict Hopf bifurcations resulting from different instability modes of the sidewall layer in the bottom half of the cylinder. The frequency $\omega_{2}$ is larger than twice the mean rotation rate of the split cylinder, and does not drive inertial waves, but $\omega_{1}$ is smaller and does drive inertial waves along a cone with cone angle $\beta=\cos ^{-1} \omega_{1}$, that is clearly evident in the $\eta$ contours. In the second case, QP2, the frequency $\omega_{1}$ component of the quasiperiodic flow has undergone a period-doubling bifurcation, and the flow now has a dominant frequency at $0.5 \omega_{1}$, which is evident in the accompanying power spectral density of the kinetic energy. Now the flow has inertial waves driven along two distinct cones, one with cone angle $\beta=\cos ^{-1} \omega_{1}$ and the other with $\beta=\cos ^{-1} 0.5 \omega_{1}$. In the third example shown in the figure, QP4, a second period-doubling bifurcation has resulted in a quasiperiodic state with strong spectral signals at frequency $0.25 \omega_{1}$ and its harmonics. In this example, $\omega_{2} \approx 2.25 \omega_{1}$ and the power spectral density is very clean. The flow has inertial waves driven along four cones with angles $\beta=\cos ^{-1} 0.25 j \omega_{1}$, $j \in[1,4]$, as shown in the $\eta$ contours.

As in [23], when the flow is not restricted to being axisymmetric, [22] have shown that the primary instabilities are three-dimensional. Depending on $\omega_{0}$ and $\delta \omega$, rotating 
(a)

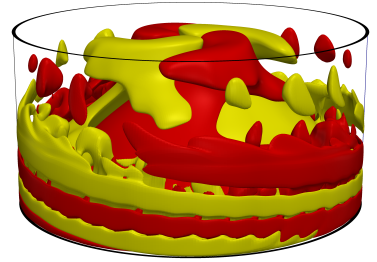

(b)

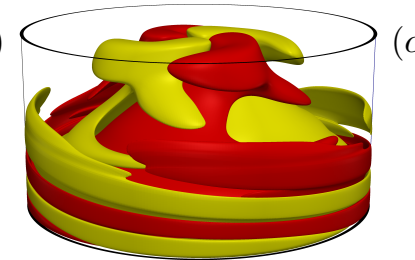

(c)

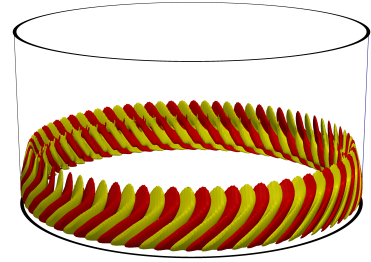

Figure 4. Isosurfaces of $(a) w-w_{0},(b) w_{2}$ and $(c) w_{39}$ for $\mathrm{QP}_{2,39}$ at $\omega_{0}=1.5 \times 10^{4}, \delta \omega=0.28$ and $\Gamma=1$; the isosurfaces are at \pm 0.001 (from [22]).

waves localised in the junction region of the slower rotating half of the cylinder are found, either with low or high azimuthal wave numbers. Figure 3 shows isosurfaces of the axial velocity perturbation, $w-w_{0}$ (i.e. the axial velocity of the full solution minus its azimuthal mean) of four low azimuthal wave number rotating waves (with azimuthal wave numbers $m \in[1,4]$ ) at the same point in parameter space. All are stable, having bifurcated in successive symmetry-breaking Hopf bifurcations within an Eckhaus band of stable azimuthal wave numbers. The rotating spiral disturbances in the junction region associated with these rotating waves provide a localised periodic disturbance which can drive wave beams into the interior if the disturbance frequency is appropriate. All four rotating waves have precession frequencies in the frame of reference rotating with the mean cylinder rotation rate such that their rotation is retrograde, and the associated frequencies are less than twice the cylinder mean rotation rate. For the lowest $m=1$, the cone angle is such that the wave beam almost perfectly retraces itself as it is emitted from the juction region $(r, z)=(1,-0.5 \Gamma)$ and is reflected back from the axis at the top endwall $(r, z)=(0,0.5 \Gamma)$. For increasing $m$, the wave beams do not penetrate all the way to the axis; for $\mathrm{RW}_{4}$, there is a small quiescent zone near the axis. This is consistent with the analysis of [44], who showed that the radial extent of the quiescent axial zone increases with $m$.

Typically however, over a wide range in $\omega_{0}$ and $\delta \omega$, quasiperiodic states that are mixed modes of high and low wave number rotating waves are found. Figure 4 shows one such case, $\mathrm{QP}_{2,39}$, which is a mix-mode consisting of rotating waves with $m=2$ and $m=39$; the perturbation axial velocity together with the $m=2$ and $m=39$ azimuthal components of the axial velocity are shown. The instabilities in the split cylinder system are complicated by having an Eckhaus band of wave number associated with them [27], and for lower $\delta \omega$ the instabilities are subcritical with an extensive region of hysteresis. The instability to low azimuthal wave numbers leads to rotating waves consisting of spiral vortex structures of alternating sign that are roughly aligned with the mean flow direction in the slower junction region. Their structure and flow alignment are suggestive of a centrifugal instability as they are akin to Görtler vortices. The high azimuthal wave number instabilities occur for lower $\delta \omega$ and are subcritical. The resulting flows consist of a large number of pairs of counter-rotating vortical structures that are aligned orthogonally to the mean flow direction in the junction region. Their structure and flow alignment are suggestive of Tollmien-Schlichting waves in the side wall and junction region closest to the wall. The precession frequencies (viewed in a frame of reference rotating at the cylinder's mean rotation rate), are in the range of 1.4 to 1.8 times the mean rotation rate, so that inertial waves would be emitted into the interior at cone angles in the range $22^{\circ}$ 


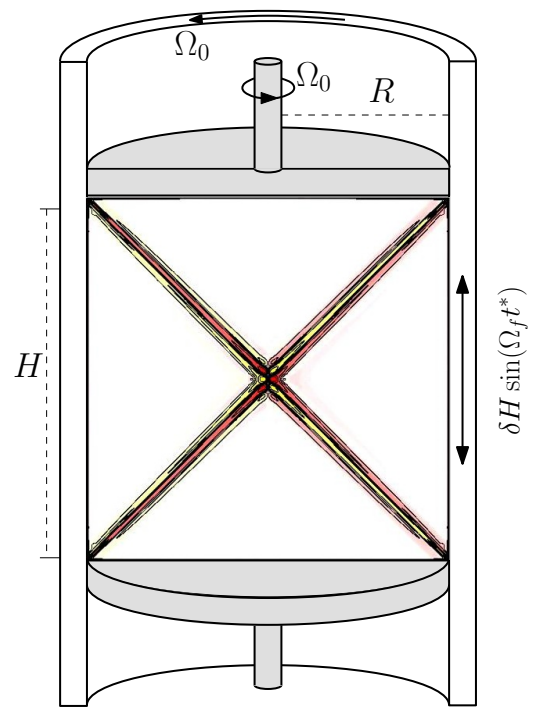

Figure 5. Schematic of the oscillatory sidewall driven flow. The insert shows azimuthal vorticity $\eta$ at one instant in time, with intense inertial wave beams which focus at the axis, for $\omega_{0}=10^{6}, \Gamma=2, A_{f}=10^{2}$ and $\cos \beta=F=$ $\Omega_{f} /\left(2 \Omega_{0}\right)=1 / \sqrt{2}$ (from [25]).

to $44^{\circ}$. However, the associated wave beams are not evident in the nonlinear solutions. High wave number beam do not penetrate deep into the interior [44, 22], and so even if there is turbulence in the boundary layers (broad-band spatial spectrum), only the low wave number part of the spectrum drives beams into the interior. So, the interior remains laminar and coherent with low wave number shear layers.

\subsection{Harmonic forcing}

The steady differential rotation discussed above can lead to inertial waves due to localised instabilities, but we have little control over what types of inertial wave response will ensue. We can explore to what extent the linear inviscid theory of rapidly rotating flows is applicable to confined systems with finite viscosity and nonlinear forcing by subjecting the confined flow to harmonic forcing with forcing frequencies less than twice the cylinder rotation rate.

In this overview, we focus our attention on three geometrically simple flows which are described in the following sections.

4.2.1. Forced sidewall oscillations We begin by describing the harmonically forced system considered in [25] that consists of a rapidly rotating cylinder whose sidewall is also oscillating harmonically in the axial direction. This forcing is attractive as the body force is simply Coriolis, $\boldsymbol{b}=\left(0,0,-2 \omega_{0}\right) \times \boldsymbol{v}=2 \omega_{0}(v,-u, 0)$. The governing equations in the rotating frame are (1) with this body force and boundary conditions

$$
\begin{aligned}
& \boldsymbol{v}=\left(0,0, A_{f} \sin \omega_{f} t\right) \text { at the sidewall } r=1, \\
& \boldsymbol{v}=(0,0,0) \text { at the top and bottom endwalls } z= \pm \Gamma / 2,
\end{aligned}
$$




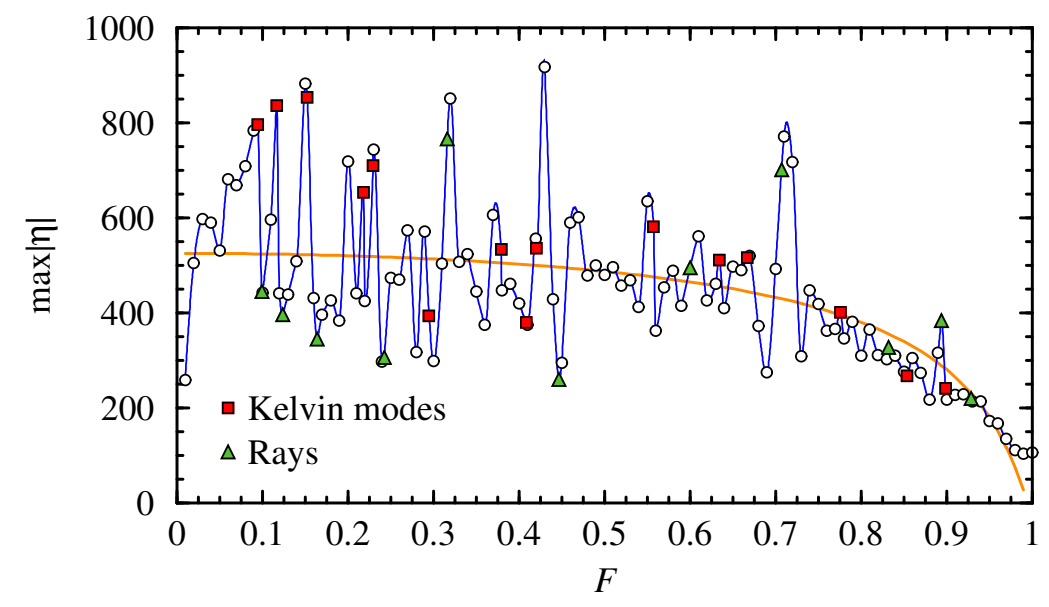

Figure 6. Response diagram in the oscillatory sidewall driven flow: open symbols are the maximum values of $|\eta|$ in the whole domain excluding the boundary layers, as a function of $F$, for $\omega_{0}=10^{5}, \Gamma=2$ and $A_{f}=10^{2}$. The blue line is a spline fit to guide the eye. The orange line is the theoretical value of $|\eta|$ given by (26). The red squares correspond to the $F$ values of the low-order inviscid Kelvin modes and the green triangles correspond to the $F$ values of the low-order retracing rays (from [25]).

where $A_{f}=\delta H \Omega_{f} R / \nu$ and $\omega_{f}=\Omega_{f} R^{2} / \nu$ are the non-dimensional amplitude and frequency of the harmonic forcing. This harmonic forcing drives an oscillatory Stokes layer in the sidewall without affecting the rotation vector of the system. For $\omega_{f}<2 \omega_{0}$, conical wave beams are emitted from the junctions between the sidewall and the endwalls, with the cone angle determined by the inertial wave dispersion relation. Figure 5 shows a schematic of this set-up, with $\omega_{f}$ and $\Gamma$ chosen to produce a retracing conical wave beam whose cross-section in a meridional plane has the form of a St. Andrew's cross.

The oscillatory Stokes boundary layer is robust to large perturbations over a very wide range of parameter space, and so this allows one to study the properties of inertial wave beams in a physically realisable system that is not overly subjected to boundary layer instabilities. A detailed numerical and theoretical analysis of the internal shear layers was presented in [25], who showed that the system losses stability to complicated three-dimensional flow when $A_{f}$ is very large, corresponding to sidewall oscillation displacement amplitudes $\delta H$ of the order of the cylinder radius. This is far removed from the displacement amplitudes of interest here, and there is a large range of governing parameters which are physically realisable in experiments in which the inertial waves are robust. This is in contrast to many other physical realisations of inertial waves where the driving mechanisms tend to lead to instabilities and complicate the study of the waves. The response diagram of the system for a large range of forcing frequencies was computed and compared with the results with inviscid eigenmodes and ray tracing techniques; see figure 6 .

According to [9], inertial waves decay exponentially with the spin-up time, which in terms of our non-dimensional variables is given by $\omega_{0}^{-1 / 2}$. If $t_{1}$ is the time taken by the inertial wave to travel a typical length $L$ (we will take $L$ to be the radius, of non-dimensional length one), we then expect a decay of the form $\exp \left(-g \omega_{0}^{1 / 2} t_{1}\right)$, 


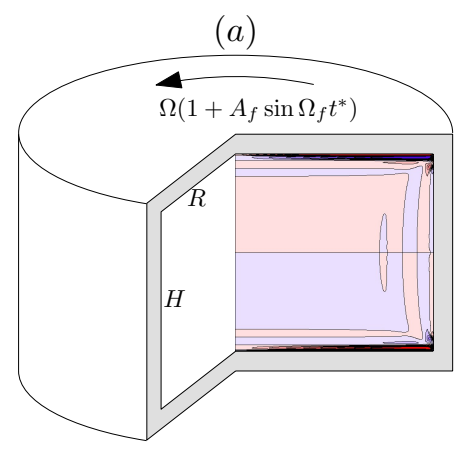

(b)

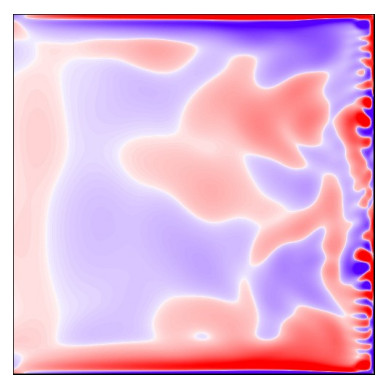

(c)

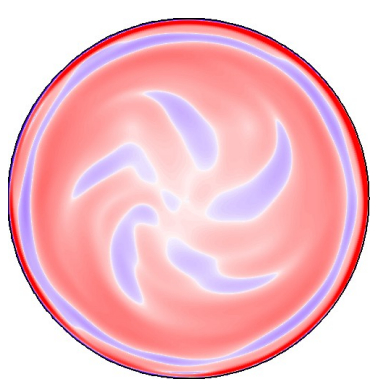

Figure 7. (a) Schematic of the libration flow. Snap-shots of $\eta$ in $(b)$ a meridional $(r, z)$-plane and $(c)$ a horizontal plane at 3/4 height for a weakly turbulent state dominated by inertial waves in the interior when $\omega_{0}=10^{4}, \Gamma=1, A_{f}=0.7$ and $\Omega_{f} / 2 \Omega_{0}=0.942$ (from [24]).

where $g$ is some $O(1)$ constant. We can estimate $t_{1}=L / c_{g}$, where $c_{g}$ is the group velocity of the inertial wave, given by $c_{g}=\left(2 \omega_{0} / k\right) \sin \beta$, from $(5)$. We also need an estimate of the wave-vector modulus $k=2 \pi / \lambda$, where $\lambda$ is the wavelength. In this problem, $\lambda$ scales as $\lambda \propto \omega_{0}^{-1 / 3}$ [25]. Putting everything together, we arrive at an estimate for the attenuation rate of the inertial waves:

$$
\psi=\psi_{0} \exp \left[-g \omega_{0}^{-1 / 6}(\sin \beta)^{-1}\right],
$$

On average, the values of $\max |\eta|$, the maximum of $|\eta|$ in the whole domain excluding the boundary layers, closely follow this expression. The best fit to this decay rate is shown in the figure as the thick orange monotonic line, given by

$$
\max |\eta|=g_{1} \exp \left[-g_{2} \omega_{0}^{-1 / 6}\left(1-F^{2}\right)^{-1 / 2}\right],
$$

with $g_{1}=852.2$ and $g_{2}=3.295$. This result complements the viscous attenuation rates given in section $\S 3$, that considered the influence of large wave numbers. Here we give the decay rate dependence on the forcing frequency, while the wave number $k$ is given by the solid body rotation, $\omega_{0}$. The response diagram shows significant departures from the mean decay rate (orange line), which can be associated with resonances and nonlinear focusing of low-order Kelvin modes and retracing wave beams.

4.2.2. Libration A harmonic forcing of rotating flows which is widely studied is libration [20]. In the context of rotating cylinder flows, libration forcing simply amounts to a modulation of the cylinder rotation rate. In the laboratory frame, the flow is governed by (1) with $\boldsymbol{b}=\mathbf{0}$ and boundary conditions

$$
\begin{aligned}
& \boldsymbol{v}=\left(0, \omega_{0}\left(1+A_{f} \sin \omega_{f} t\right), 0\right) \text { at } r=1, \\
& \boldsymbol{v}=\left(0, r \omega_{0}\left(1+A_{f} \sin \omega_{f} t\right), 0\right) \text { at } z= \pm \Gamma / 2,
\end{aligned}
$$

where $A_{f}$ and $\omega_{f}$ are the non-dimensional amplitude and frequency of the modulation of the solid body rotation $\boldsymbol{v}=\left(0, r \omega_{0}, 0\right)$, where $\omega_{0}=\Omega_{0} R^{2} / \nu$. A schematic of the flow is show in figure $7(a)$.

For small forcing amplitudes $A_{f}$, the unsteady boundary layer profiles at the top and bottom endwalls have two length scales, given by $\delta_{ \pm}=\sqrt{2 /\left|\omega_{f} \pm 2 \omega_{0}\right|}$. In 
dimensional terms, $\delta_{ \pm}^{*}=\sqrt{2 \nu /\left|\Omega_{f} \pm 2 \Omega_{0}\right|}$ [43]. This scaling is very different from the typical Ekman scaling, $\delta^{*}=\sqrt{\nu / \Omega_{0}}$, used to analyse boundary layers in rotating flows and used to introduce viscous corrections to the inviscid theory [10]. The endwall layers in librating cylinder flows are a combination of Ekman and oscillatory Stokes boundary layers [24]. The scaling depends explicitely on the forcing frequency, and when $\Omega_{f}$ approaches $2 \Omega_{0}$, i.e. when the linear inviscid equation (2) switches between being hyperbolic and elliptic, $\delta_{-}$becomes unbounded: the boundary layers fill the whole domain and the boundary layer concept loses its meaning. The sidewall boundary layer is more complicated than the endwall boundary layers, comprised of several nested layers [43], and as the forcing amplitude is increased, they undergo instabilities that are centrifugal in nature and manifest most strongly during the retrograde phase of the harmonic forcing [24].

Under libration forcing, the bulk of the fluid is no longer in solid body rotation. The forcing not only drives a mean streaming azimuthal (zonal) flow, but also a mean meridional flow. The azimuthal streaming flow is retrograde and scales quadratically with the amplitude of the forcing [24]. It has drawn much attention [41, 5, 4, 36], whilst the meridional mean flow has not. The meridional mean flow can have important dynamic consequences, such as the quenching of wall modes in modulated rotating convection [37].

Salient features of the libration flow include: $(i)$ sidewall boundary layer instabilities that can generate inertial waves even in frequency regimes where $\Omega_{f}>2 \Omega_{0}$; (ii) modulation-driven inertial waves that undergo symmetry-breaking bifurcations in regimes where there are no sidewall instabilities, leading to complex three-dimensional interior flows; (iii) three-dimensional interior inertial wave flows that exhibit heteroclinic dynamics and Eckhaus instabilities with very long time scales (of the order of the viscous time); and (iv) complicated interactions between the threedimensional inertial waves and the boundary layers leading to fully turbulent states. The current state-of-the-art experiments of these flows [36] have only been able to use flow visualisation to observe the instabilities and turbulence in the boundary layers, and time-averaged measurements of the interior flow which lack the necessary temporal resolution to interrogate the details of the three-dimensional inertial waves in the interior.

The numerical study of the librating cylinder flows in [24] incorporates the full three-dimensional Navier-Stokes equations with the physical no slip boundary conditions. The librating cylinder flow provides one of the few clear examples of inertial wave states undergoing a sequence of bifurcations as the driving force is increased: Hopf bifurcations of limit cycles, Eckhaus exchange of azimuthal wave number states, heteroclinic cycle dynamics leading to chaotic flow or weak turbulence as stable and unstable manifolds intersect transversely in phase space (see figure 7 for an example showing such a turbulent state). A complication with librating flows is that the mechanism that drives the inertial waves is also driving a centrifugal instability of the sidewall boundary layer during the deceleration (retrograde) phase of the libration and this makes it difficult (if not impossible) to separate which mechanism is responsible for the transition to turbulence.

4.2.3. Precession Precessing flows are rapidly rotating flows with the container rotation axis precessing around another fixed rotation axis. These flows are common in many geophysical and astrophysical settings [20]. Figure 8 shows a schematic of the 


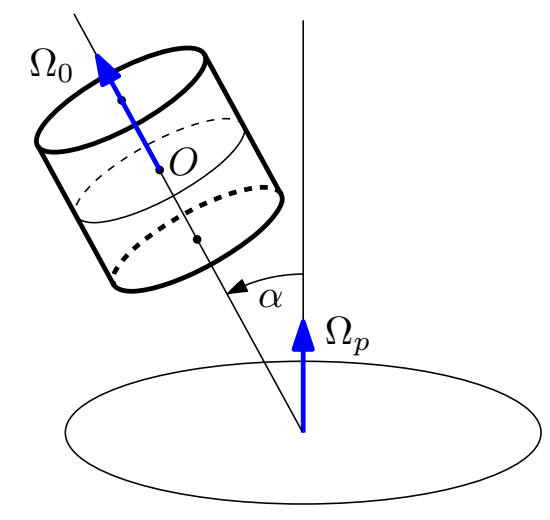

Figure 8. Schematic of the precessing cylinder.

precessionaly forced rotating cylinder; the cylinder rotates around its axis with angular velocity $\Omega_{0}$, which is fixed in a rotating table with angular velocity $\Omega_{p}$. The nutation angle between the two axes is $\alpha$. The governing equations are (1) in a reference system rotating with $\Omega_{p}$ (the rotating table reference frame), with body force $\boldsymbol{b}=-2 \boldsymbol{\omega}_{p} \times \boldsymbol{v}$ and boundary conditions $\boldsymbol{v}=\left(0, r \omega_{0}, 0\right)$, where $\omega_{p}=\Omega_{p} R^{2} / \nu$ and $\omega_{0}=\Omega_{0} R^{2} / \nu$. The body force is steady in this reference frame, and has azimuthal wave number $m=1$ spatial structure. This body force results in a base state resembling the $(1,1,1)$-Kelvin mode, referred to as the spin-over flow.

There have been many experimental studies on precessing cylinder flows [30, 31, $32,16,17]$. More recent experiments include quantitative measurements focusing on triadic resonances $[34,18,2]$. Triadic resonances between the $(1,1,1)$-Kelvin mode and two additional modes $\left(k_{1}, m_{1}, n_{1}\right)$ and $\left(k_{2}, m_{2}, n_{2}\right)$ are possible when $\left|n_{2} \pm n_{1}\right|=1$, $\left|m_{2} \pm m_{1}\right|=1$, and $\left|\sigma_{k_{2}, m_{2}, n_{2}} \pm \sigma_{k_{1}, m_{1}, n_{1}}\right|=\omega_{0}[18,19]$. The ratio $\sigma_{k, m, n} / \omega_{0}$ depends on the nutation angle $\alpha$, the aspect ratio $\Gamma=H / R$ and the frequency ratio $\omega_{p} / \omega_{0}$. For exact 1:5:6 resonance, the resonance condition is

$$
\frac{\sigma_{1,1,1}}{\omega_{0}}=\frac{\sigma_{1,-5,1}}{\omega_{0}}+\frac{\sigma_{1,6,2}}{\omega_{0}}=1,
$$

and only one free parameter is left. For exemple, at $\alpha_{0}=1^{\circ}$, the resonance condition requires $\omega_{p} / \omega_{0}=-0.15253$ and $\Gamma=1.62$. In order to explore this triadic resonance, the geometry is fixed and $\omega_{p}$ and $\omega_{0}$ must be varied in such a way that their ratio remains constant. If we change any other parameter (for example one of the frequencies, or the nutation angle $\alpha$ ), the system is detuned away from resonance:

$$
\frac{\sigma_{1,1,1}}{\omega_{0}}=\frac{\sigma_{1,-5,1}}{\omega_{0}}+\frac{\sigma_{1,6,2}}{\omega_{0}}=\frac{\omega_{0}+\omega_{p} \cos \alpha}{\omega_{0}+\omega_{p} \cos \alpha_{0}}=1+\delta,
$$

where $\delta$ is the detuning parameter.

The 1:5:6 resonance at $\alpha_{0}=1^{\circ}, \omega_{p} / \omega_{0}=-0.15253$ and $\Gamma=1.62$, has been numerically explored in detail in [33]. Increasing $\omega_{0}$, i.e. reducing the influence of viscosity in the system, [33] have found that the base state BS, corresponding to the forced spin-over flow with azimuthal wave number $m=1$, is stable up to $\omega_{0} \approx 4777$. For smaller $\omega_{0}$ viscous effects dominate the flow. Above the critical $\omega_{0}$ value, the triadic resonance comes into play. The first bifurcation is a Hopf bifurcation where the resonant modes with azimuthal wave numbers $m=5$ and $m=6$ bifurcate from the 


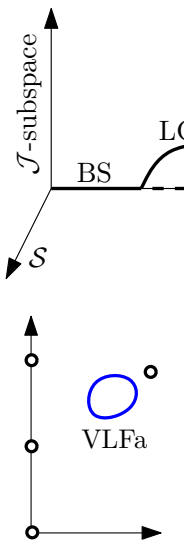

$\omega_{0}=7050$

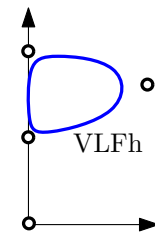

$\omega_{0}=7150$

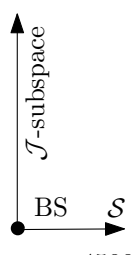

$\omega_{0}=4500$
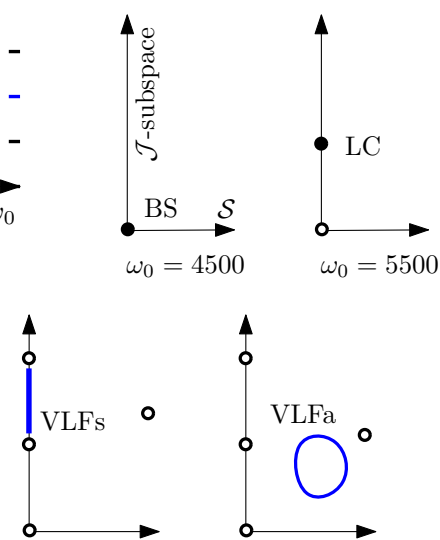

$\omega_{0}=7200$

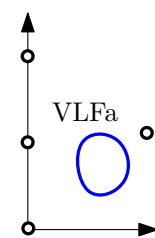

$\omega_{0}=7250$
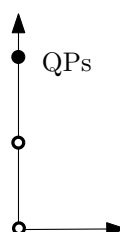

$\omega_{0}=6300$

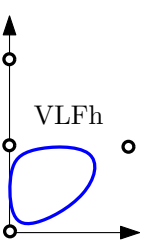

$\omega_{0}=7400$

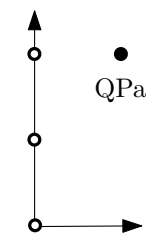

$\omega_{0}=6500$

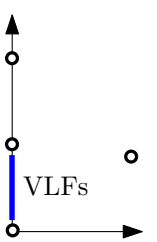

$\omega_{0}=7670$

Figure 9. Schematic bifurcation diagram and selected phase portraits of the different solutions obtained. The states BS, LC and QPs are all in the $\mathcal{I}$-symmetric subspace $(S=0)$, while $\mathrm{QPa}$ and most of the VLF states are not $(S \neq 0)$. Stable states are shown as filled circles and thick lines, while unstable states are open circles (from [33]).

base state, both with the same frequency $\omega_{1}$, in agreement with the triadic-resonance theory. The result is a limit cycle LC whose $m=5$ and $m=6$ azimuthal components rotate in the azimuthal direction almost uniformly. On increasing $\omega_{0}$, LC loses stability at a secondary Hopf bifurcation to a two-torus state QPs. The new frequency introduced at this bifurcation, $\omega_{2}$, is smaller than but close to $\omega_{1}$ and corresponds to a radial oscillation of the streaming flow, that now becomes time dependent. The governing equations and boundary conditions are invariant under a spatial symmetry, the inversion $\mathcal{I}$, whose action on the velocity is $\mathcal{A}(\mathcal{I}) \boldsymbol{u}=(u, v,-w)(r, \theta+\pi,-z, t)$. The states described so far (BC, LC and QPs) are $\mathcal{I}$-invariant. The symmetry parameter,

$$
S=\|\boldsymbol{u}-\mathcal{A}(\mathcal{I}) \boldsymbol{u}\|_{2} .
$$

is a measure of the departure of the solutions from symmetry. For time-dependent solutions, the symmetry parameter is also time dependent and we will use its maximum value over an appropriate large time interval $S_{\mathrm{M}}=\max _{t} S(t)$. This symmetry is broken in the following bifurcation of QPs, resulting in an asymmetric two-torus QPa. This sequence of bifurcations resembles the classical Ruelle-Takens route to chaos $[38,35]$, and is illustrated in the first row of figure 9 , showing a schematic bifurcation diagram and selected schematic phase portraits of the solutions analyzed in detail in [33].

According to the Ruelle-Takens scenario, the next bifurcation would result in chaotic dynamics. What is obtained however, is different. At $\omega_{0} \approx 7025$ the two-torus QPa state bifurcates to a three-torus state with an additional very-low-frequency (VLF) $\omega_{3}$. In the range $\omega_{0} \in[7025,8000]$, a variety of VLF states are found, and schematic phase portraits of these are shown in the second row of figure 9 . The third frequency $\omega_{3}$ is associated with the exchange of energy between the resonant $m=5$ and $m=6$ flow components and the $m=0$ streaming flow component, and corresponds to slow drifts in phase space between some of the four states obtained in the first three bifurcations (BS, LC, QPs and QPa), which are now all unstable; 
(a) $\alpha=1.432^{\circ}$

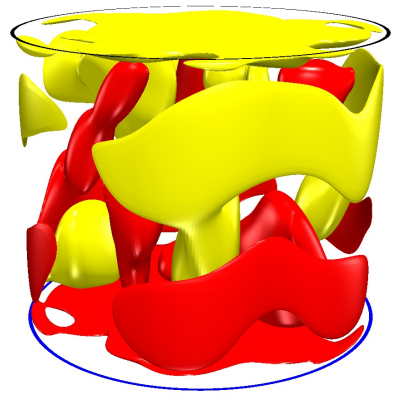

(b) $\alpha=8.6^{\circ}$

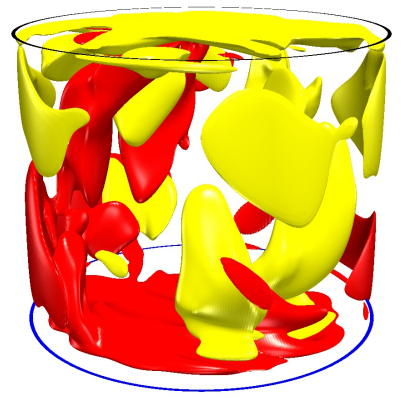

(c) $\alpha=47^{\circ}$

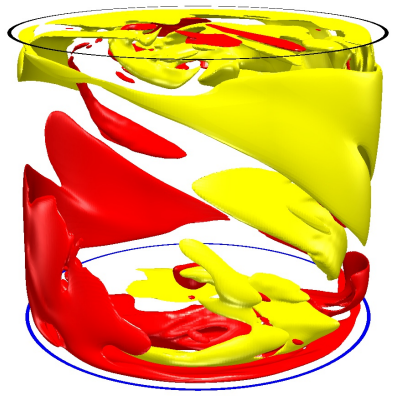

Figure 10. Helicity isosurfaces of typical states in the three $\alpha$ regimes for $\omega_{0}=4000, \omega_{p}=-610$ and $\Gamma=1.62$ (from [26]).

the drifts are close to heteroclinic cycles between some of these unstable (hyperbolic) states. Moreover, some of these drifts are into and out of the $\mathcal{I}$-symmetric subspace, as illustrated in the second row of figure 9. These VLF states are quasiperiodic three-tori or weakly chaotic states, and the heteroclinic dynamics found suggest the presence of chaotic attractors in their neighborhood [11].

While triadic resonances have been observed in precession experiments, they have only been reported for very small nutation angles, $\alpha \sim 1^{\circ}$, for which there is no transition to turbulence $[18,34,19]$. Recently, direct numerical simulations have reproduced the experimental observations, and have provided deeper insight into the nonlinear bifurcation sequences involving the triadic resonance $[1,33,2]$, but these still did not capture the to turbulence. So, it is very likely that other mechanisms for the transition are at play. The experiments clearly show a very large departure from steady axisymmetric solid body rotation on which the modes in the triadic resonance are theoretically based. A mechanism which may play a dominant role in the transition to turbulence comes from the separated boundary layers due to the constantly imposed accelerations from the precession. These separated boundary layer are unstable. In [26], the influence of the nutation angle $\alpha$ on the precessing cylinder flow was investigated, shedding some light on the sudden transitions to turbulence observed in experiments. Keeping $\Gamma=1.62, \omega_{0}=4000$ and $\omega_{p}=-610$ fixed, and varying $\alpha$ from $0.5^{\circ}$ to $47^{\circ}$, three different dynamic regimes were identified from the Navier-Stokes simulations. A typical example in each of the three regimes is shown in figure 10, showing isosurfaces of the helicity.

The main differences in the three regimes are illustrated in figure 11 in terms of the energies of the azimuthal Fourier components of the flow scaled by the kinetic energy of solid body rotation, $e_{m}$, the symmetry parameter $S_{\mathrm{M}}$, as well as the forcing amplitude $A_{f}=\left|\omega_{p} / \omega_{0}\right| \sin \alpha$ and the detuning parameter $\delta$. In the low- $\alpha$ regime $\left(\alpha \lesssim 4^{\circ}\right)$, detuning effects are negligible, the precessional forcing is weak, and the dynamics are dominated by the triadic resonance. The $m=5$ and 6 Fourier components of the flow have much larger energies than the other components with $m>1$, as predicted by the weakly nonlinear theory [19]. However, additional bifurcations leading to quasiperiodic and weakly chaotic solutions occur for small increases in $\alpha$. The inversion symmetry of the flow is broken at the bifurcation to VLFa, although the bifurcated solutions retain this $\mathcal{I}$-symmetry when averaged in time [33]. The boundary layers are very similar to the boundary layers of the steady base state solution, and the bulk of the 


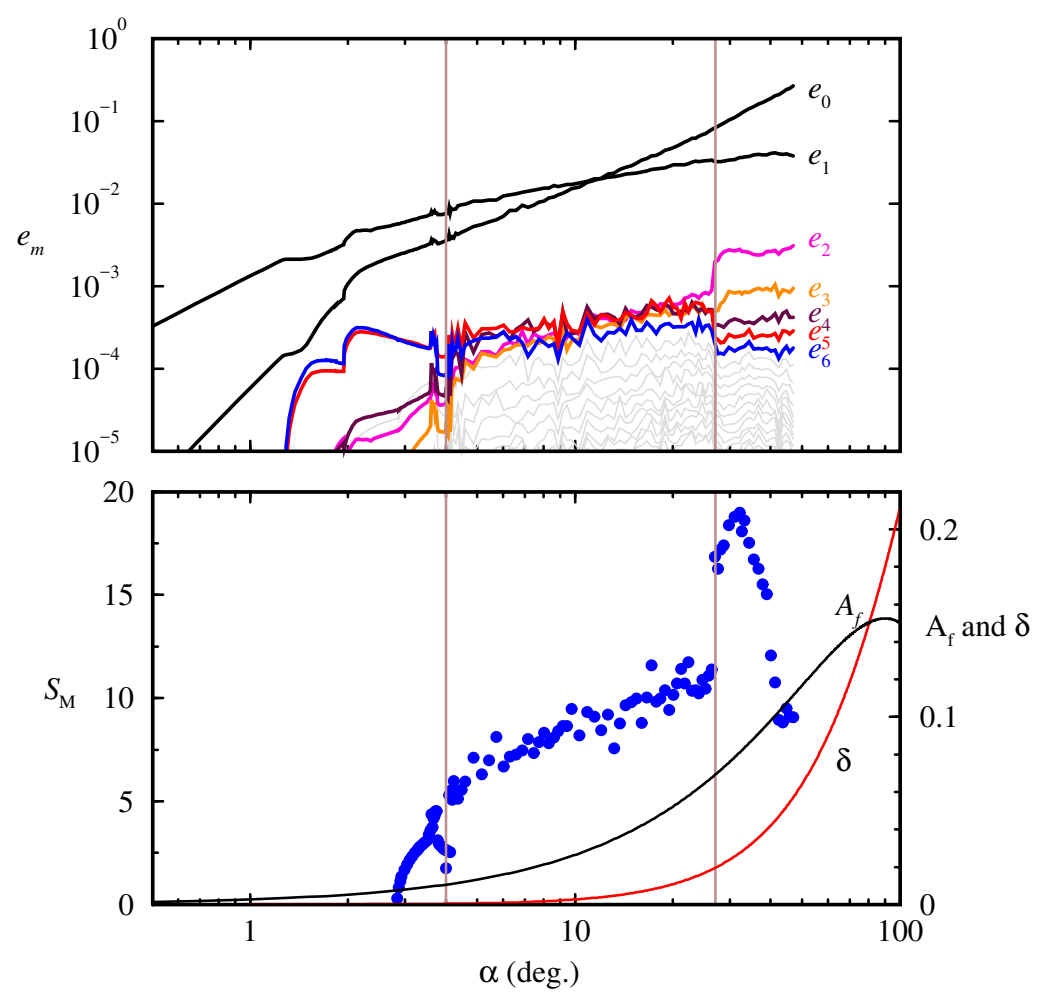

Figure 11. Variations of relative modal kinetic energies $e_{m}$ (top) and $S_{\mathrm{M}}, A_{f}$ and $\delta$ (bottom), with $\alpha$. The two vertical lines at $\alpha=4^{\circ}$ and $27^{\circ}$ demarcate three regimes of differing dynamics (from [26]).

flow is dominated by columnar vortices due to the triadic resonance mechanism.

In the mid- $\alpha$ regime $\left(4^{\circ} \lesssim \alpha \lesssim 27^{\circ}\right)$, the detuning effects are still weak, the forcing is stronger, and the dynamics are spatio-temporally complex due to nonlinear interactions between the triadic resonance driven flow components and the nonlinear harmonics of the $m=1$ overturning flow. As shown in figure 11, the energies $e_{m}$, $m \in[2,6]$, have very similar levels. This is a clear indication of the strong interaction between the triadic resonance mechanism and the nonlinear effects. The symmetry parameter $S_{\mathrm{M}}$ increases steadily with $\alpha$ in this regime. The boundary layers are still similar to the boundary layers of the base state solution, but with larger deformations, and the columnar vortices in the bulk of the flow are still present, but they are no longer uniformly distributed in azimuth, and undergo break-up and reformation in a spatio-temporally complex fashion.

In the large- $\alpha$ regime $\left(\alpha \gtrsim 27^{\circ}\right)$, the detuning is no longer negligible, and the amplitude of the forcing is larger than in lower- $\alpha$ regimes. Over a narrow interval in $\alpha$, centred at $\alpha=27^{\circ}$, the flow undergoes dramatic changes, as illustrated in figure 11 . The energies of the Fourier modes $m=2$ to 6 , that were of comparable strength in the mid- $\alpha$ regime, change abruptly: all the energies $e_{m}$ now decrease with increasing $m$, spread over more than a decade in energy levels. The Fourier components of the flow essentially become harmonics of the $m=1$ overturning flow, and the triadic resonance mechanism does not play any significant role. There is also an abrupt increase in the 
flow asymmetry: $S_{\mathrm{M}}$ almost doubles and remains very high up to $\alpha \lesssim 40^{\circ}$. This is due to asymmetric eruptions from the boundary layers, that occur erratically with time and location in the various boundary layers. The sidewall boundary layer structure is also completely different to that in the lower- $\alpha$ regimes, with an oblique plane separating the positive and negative helicity parts of the boundary layer. The flow in the interior of the cylinder is devoid of helical columns, and the only structures that are apparent are related to the boundary layer eruptions forming short-lived internal shear layers that are predominantly located near the boundary layers. Increasing the nutation angle above $40^{\circ}$ results in a decrease in the asymmetry of the flow. This is due to the boundary layer eruptions being more symmetrically distributed. The eruptions from the boundary layer take place mainly around the junctions of the endwalls and the sidewall where the overturning flow is strongest. The interior of the cylinder does not exhibit any large scale structure.

The numerical simulations presented in [26] show that the flow undergoes dramatic changes as the nutation angle $\alpha$ is increased while the remaining parameters are held fixed. The fixed parameters represent the geometry $(\Gamma)$, the cylinder and table angular velocities and the fluid viscosity $\left(\omega_{0}\right.$ and $\left.\omega_{p}\right)$. Of course, increasing $\alpha$ results in an increase in the amplitude of the forcing $A_{f}$ (because the component of the rotation orthogonal to the cylinder axis increases), and also produces a detuning away from the triadic resonance condition. The distortion of the sidewall boundary layer, with an oblique plane separating its positive and negative helical parts and the subsequent distortion of the overturning flow are associated with the flow trying to accommodate to a total angular velocity that is widely misaligned with the cylinder axis for large $\alpha$. In this high- $\alpha$ regime, $e_{0}$ is as much as 0.25 , i.e. the nonlinear mean flow modification is about $25 \%$ of the nominal solid body rotation associated with the cylinder rotation. This is a massive disruption to the solid body rotation on which the linear inviscid theory of Kelvin modes is based. So, in this sense it is not surprising that Kelvin mode triadic resonance effects are not present in the high- $\alpha$ regime.

\section{Discussion and perspectives}

Inertial waves in rapidly rotating flows are ubiquitous. They are present in some geophysical and astrophysical flows. The study of inertial waves dates back to Lord Kelvin [14]. However, they are not yet fully understood. One of the reasons is that inertial waves are quickly damped by viscosity, and are usually weak compared with other flow features. Therefore, only specifically tailored flows allow for their detailed study. The classical instability mechanisms in fluid dynamics (such as shear, thermal, centrifugal and boundary layer instabilities) compete and are intermixed with inertial wave effects. The mutual interplay between all these mechanisms is better understood in terms of dynamical systems theory, that provides the building blocks (in terms of local and global bifurcations, strange attractors, etc.) describing the transition processes between laminar flows and turbulence. We have reviewed and analysed a variety of rapidly rotating flows examining the transition processes and how these are influenced by inertial waves.

In many of these examples, the primary instabilities as the rotation rate is increased are to three-dimensional flows, often in subcritical bifurcations, and involve multiple modes in an Eckhaus band of wave numbers. These features cannot be captured in axisymmetric studies that are very commonly used in rapidly rotating flows; fully three-dimensional analysis is unavoidable. Furthermore, linear stability 
analysis is often inadequate as the flows involve heteroclinic dynamics with drifts amongst a multitude of saddle states. These dynamics can only be addressed from a fully nonlinear perspective. Rotating turbulence is inherently non-isotropic, and the effects of boundaries, regardless of how "far" away they are are ever present, and the boundary details completely dominate the "interior" dynamics.

\section{Acknowledgments}

This paper is based on an invited lecture that was presented at the Turbulent Mixing and Beyond Workshop at the Abdus Salam International Centre for Theoretical Physics (Trieste, Italy) in August 2014 by JML, who is grateful for the financial support received from the conference organisers. The research reported here was supported by US NSF Grant CBET-1336410 and Spanish MECD Grant FIS201340880-P.

\section{References}

[1] T. Albrecht, H. M. Blackburn, J. M. Lopez, R. Manasseh, and P. Meunier. Triadic resonances in precessing rapidly rotating cylinder flows. J. Fluid Mech., 778:R1, 2015.

[2] T. Albrecht, H. M. Blackburn, P. Meunier, R. Manasseh, and J. M. Lopez. Experimental and numerical investigation of a strongly-forced precessing cylinder flow. Int. J. Heat 85 Fluid Flow, 2016. http://dx.doi.org/10.1016/j.ijheatfluidflow.2016.05.009.

[3] G. K. Batchelor. An Introduction to Fluid Mechanics. Cambridge University Press, 1967.

[4] F. H. Busse. Mean zonal flows generated by librations of a rotating spherical cavity. J. Fluid Mech., 650:505-512, 2010 .

[5] F. H. Busse. Zonal flow induced by longitudinal librations of a rotating cylindrical cavity. Physica D, 240:208-211, 2010.

[6] P. Chossat and R. Lauterbach. Methods in Equivariant Bifurcations and Dynamical Systems. World Scientific, 2000.

[7] P.-P. Cortet, C. Lamriben, and F. Moisy. Viscous spreading of an inertial wave beam in a rotating fluid. Phys. Fluids, 22:086603, 2010.

[8] M. Duran-Matute, J.-B. Flór, F. S. Godeferd, and C. Jause-Labert. Turbulence and columnar vortex formation through inertial-wave focusing. Phys. Rev. E, 87:041001(R), 2013.

[9] H. P. Greenspan. On the general theory of contained rotating fluid motions. J. Fluid Mech., 22:449-462, 1965.

[10] H. P. Greenspan. The Theory of Rotating Fluids. Cambridge University Press, 1968.

[11] J. Guckenheimer, B. Krauskopf, H. M. Osinga, and B. Sandstede. Invariant manifolds and global bifurcations. Chaos, 25:097604, 2015.

[12] P. Gutierrez-Castillo and J. M. Lopez. Instabilities of the sidewall boundary layer in a rapidly rotating split cylinder. Eur. J. Mech. B-Fluids, 52:76-84, 2015.

[13] J. E. Hart and S. Kittelman. Instabilities of the sidewall boundary layer in a differentially driven rotating cylinder. Phys. Fluids, 8:692-696, 1996.

[14] Lord Kelvin. Vibrations of a columnar vortex. Phil. Mag., 10:155-168, 1880.

[15] R. R. Kerswell. Elliptical instability. Ann. Rev. Fluid Mech., 34:83-113, 2002.

[16] J. J. Kobine. Inertial wave dynamics in a rotating and precessing cylinder. J. Fluid Mech., 303:233-252, 1995

[17] J. J. Kobine. Azimuthal flow associated with inertial wave resonance in a precessing cylinder. J. Fluid Mech., 319:387-406, 1996.

[18] R. Lagrange, C. Eloy, F. Nadal, and P. Meunier. Instability of a fluid inside a precessing cylinder. Phys. Fluids, 20:081701, 2008.

[19] R. Lagrange, P. Meunier, F. Nadal, and C. Eloy. Precessional instability of a fluid cylinder. J. Fluid Mech., 666:104-145, 2011.

[20] M. Le Bars, D. Cebron, and P. Le Gal. Flows driven by libration, precession, and tides. Ann. Rev. Fluid Mech., 47:163-193, 2015.

[21] J. M. Lopez. Characteristics of endwall and sidewall boundary layers in a rotating cylinder with a differentially rotating endwall. J. Fluid Mech., 359:49-79, 1998. 
[22] J. M. Lopez and P. Guitierrez-Castillo. Three-dimensional instabilities and inertial waves in a rapidly rotating split-cylinder flow. J. Fluid Mech., 800:666-687, 2016.

[23] J. M. Lopez and F. Marques. Sidewall boundary layer instabilities in a rapidly rotating cylinder driven by a differentially co-rotating lid. Phys. Fluids, 22:114109, 2010.

[24] J. M. Lopez and F. Marques. Instabilities and inertial waves generated in a librating cylinder. J. Fluid Mech., 687:171-193, 2011.

[25] J. M. Lopez and F. Marques. Rapidly rotating cylinder flow with an oscillating sidewall. Phys. Rev. E, 89:013019, 2014.

[26] J. M. Lopez and F. Marques. Nonlinear and detuning effects of the nutation angle in precessionally-forced rotating cylinder flow. Phys. Rev. Fluids, 1:023602, 2016.

[27] J. M. Lopez, F. Marques, I. Mercader, and O. Batiste. Onset of convection in a moderate aspect-ratio rotating cylinder: Eckhaus-Benjamin-Feir instability. J. Fluid Mech., 590:187208, 2007.

[28] L. R. M. Maas. Wave focusing and ensuing mean flow due to symmetry breaking in rotating fluids. J. Fluid Mech., 437:13-28, 2001.

[29] N. Machicoane, P.-P. Cortet, B. Voisin, and F. Moisy. Influence of the multipole order of the source on the decay of an inertial wave beam in a rotating fluid. Phys. Fluids, 27:066602, 2015 .

[30] R. Manasseh. Breakdown regimes of inertia waves in a precessing cylinder. J. Fluid Mech., 243:261-296, 1992.

[31] R. Manasseh. Distortions of inertia waves in a rotating fluid cylinder forced near its fundamental mode resonance. J. Fluid Mech., 265:345-370, 1994.

[32] R. Manasseh. Nonlinear behaviour of contained inertia waves. J. Fluid Mech., 315:151-173, 1996.

[33] F. Marques and J. M. Lopez. Precession of a rapidly rotating cylinder flow: traverse through resonance. J. Fluid Mech., 782:63-98, 2015.

[34] P. Meunier, C. Eloy, R. Lagrange, and F. Nadal. A rotating fluid cylinder subject to weak precession. J. Fluid Mech., 599:405-440, 2008.

[35] S. Newhouse, D. Ruelle, and F. Takens. Occurrence of strange axiom-A attractors near quasiperiodic flows on $T^{m}, m \geq 3$. Commun. Math. Phys., 64:35, 1978.

[36] J. Noir, M. A. Calkins, J. Cantwell, and J. M. Aurnou. Experimental study of libration-driven zonal flows in a straight cylinder. Phys. Earth Planetary Int., 182:98-106, 2010.

[37] A. Rubio, J. M. Lopez, and F. Marques. Interacting oscillatory boundary layers and wall modes in modulated rotating convection. J. Fluid Mech., 625:75-96, 2009.

[38] D. Ruelle and F. Takens. On the nature of turbulence. Commun. Math. Phys., 20:167, 1971.

[39] J. Serrin. On the stability of viscous fluid motion. Arch. Rat. Mech. Anal., 3:1-13, 1959.

[40] K. Stewartson. On almost rigid rotations. J. Fluid Mech., 3:17-26, 1957.

[41] A. Tilgner. Zonal wind driven by inertial modes. Phys. Rev. Lett., 99:194501, 2007.

[42] G. J. F. van Heijst. The shear-layer structure in a rotating fluid near a differentially rotating sidewall. J. Fluid Mech., 130:1-12, 1983.

[43] C.-Y. Wang. Cylindrical tank of fluid oscillating about a state of steady rotation. J. Fluid Mech., 41:581-592, 1970.

[44] W. W. Wood. Inertial modes with large azimuthal wavenumbers in an axisymmetric container. J. Fluid Mech., 105:427-449, 1981.

[45] K. Zhang and X. Liao. On the initial-value problem in a rotating circular cylinder. J. Fluid Mech., 610:425-443, 2008. 